\title{
EXISTENCE AND NONEXISTENCE OF POSITIVE SOLUTIONS FOR QUASILINEAR SYSTEMS
}

\author{
HAIYAN WANG
}

Received 14 October 2005; Revised 6 February 2006; Accepted 14 February 2006

The paper deals with the existence and nonexistence of positive solutions for a class of $p$-Laplacian systems. We investigate the effect of the size of the domain on the existence of positive solution for the problem in sublinear cases. We will use fixed point theorems in a cone.

Copyright (c) 2006 Haiyan Wang. This is an open access article distributed under the Creative Commons Attribution License, which permits unrestricted use, distribution, and reproduction in any medium, provided the original work is properly cited.

\section{Introduction}

In this paper we consider the existence and nonexistence of positive solutions to the boundary value problem of the $p$-Laplacian system

$$
\begin{gathered}
\left(t^{N-1}\left|u_{i}^{\prime}(t)\right|^{p-2} u_{i}^{\prime}(t)\right)^{\prime}+t^{N-1} f_{i}\left(u_{1}, \ldots, u_{n}\right)=0, \quad 0<t<R, i=1, \ldots, n, \\
u_{i}^{\prime}(0)=u_{i}(R)=0, \quad i=1, \ldots, n,
\end{gathered}
$$

where $p>1, N \geq 1, R>0$, and $f_{i}$ is nonnegative continuous, $i=1, \ldots, n$.

Such a problem arises when we seek the radial solutions of the following elliptic system:

$$
\begin{gathered}
-\Delta_{p} u_{i}=f_{i}\left(u_{1}, \ldots, u_{n}\right) \quad \text { in } B, i=1, \ldots, n, \\
u_{i}=0 \quad \text { on } \partial \Omega, i=1, \ldots, n,
\end{gathered}
$$

where $\Delta_{p} u_{i}=\operatorname{div}\left(\left|\nabla u_{i}\right|^{p-2} \nabla u_{i}\right), i=1, \ldots, n, p>1, B=\left\{x \in \mathbb{R}^{N}:|x|<R\right\}, R>0$.

Equation (1.2) covers several important cases. When $p=2$, (1.2) becomes the elliptic system

$$
\begin{gathered}
-\Delta u_{i}=f_{i}\left(u_{1}, \ldots, u_{n}\right) \quad \text { in } B, i=1, \ldots, n, \\
u_{i}=0 \quad \text { on } \partial B, i=1, \ldots, n .
\end{gathered}
$$


2 Existence and nonexistence of positive solutions

When $n=1,(1.2)$ becomes the usual $p$-Laplacian

$$
\begin{gathered}
-\Delta_{p} u=f(u) \text { in } B, \\
u=0 \quad \text { on } \partial B .
\end{gathered}
$$

When $n=1$ and $p=2$, (1.2) becomes the usual Laplacian

$$
\begin{gathered}
-\Delta u=f(u) \quad \text { in } B \\
u=0 \quad \text { on } \partial B .
\end{gathered}
$$

In several papers $[6,8]$, Wang studied the existence of nontrivial solutions of (1.1) for a fixed $R>0$. It was shown that (1.1), for a fixed $R>0$, has a nontrivial solution for sublinear nonlinearities. Related results can also be found in [1].

In this paper we investigate the effect of the size of the domain on the existence and nonexistence of positive solutions of the quasilinear elliptic system (1.1) in sublinear cases.

Let $\mathbb{R}=(-\infty, \infty), \mathbb{R}_{+}=[0, \infty)$, and $\mathbb{R}_{+}^{n}=\prod_{i=1}^{n} \mathbb{R}^{+}$. Also, for $\mathbf{u}=\left(u_{1}, \ldots, u_{n}\right) \in \mathbb{R}_{+}^{n}$, let $\|\mathbf{u}\|=\sum_{i=1}^{n}\left|u_{i}\right|$ and

$$
\mathbf{f}(\mathbf{u})=\left(f_{1}(\mathbf{u}), \ldots, f_{n}(\mathbf{u})\right)=\left(f_{1}\left(u_{1}, \ldots, u_{n}\right), \ldots, f_{n}\left(u_{1}, \ldots, u_{n}\right)\right) .
$$

We now turn to the general assumptions for this paper.

(H1) $f_{i}: \mathbb{R}_{+}^{n} \rightarrow \mathbb{R}_{+}$is continuous, $i=1, \ldots, n$.

(H2) There exists an $i \in\{1, \ldots, n\}$ such that

$$
\lim _{\|\mathbf{u}\| \rightarrow 0} \frac{f_{i}(\mathbf{u})}{\|\mathbf{u}\|^{p-1}}=\infty
$$

for $\mathbf{u}=\left(u_{1}, \ldots, u_{n}\right) \in \mathbb{R}_{+}^{n}$.

(H3) For all $i \in\{1, \ldots, n\}$,

$$
\lim _{\|\mathbf{u}\| \rightarrow \infty} \frac{f_{i}(\mathbf{u})}{\|\mathbf{u}\|^{p-1}}=0
$$

where $\mathbf{u}=\left(u_{1}, \ldots, u_{n}\right) \in \mathbb{R}_{+}^{n}$.

The main results of this paper are Theorems 1.1, 1.2, and 1.3.

Theorem 1.1. Assume (H1) and (H2) hold. Then there is an $R_{0}>0$ such that (1.1) has a positive solution for $0<R<R_{0}$.

Theorem 1.2. Assume (H1), (H2), and (H3) hold. Then (1.1) has a positive solution for all $R>0$.

The following assumption will allow us to establish a nonexistence theorem.

(H4) For all $i \in\{1, \ldots, n\}$,

$$
\limsup _{\|\mathbf{u}\| \rightarrow 0} \frac{f_{i}(\mathbf{u})}{\|\mathbf{u}\|^{p-1}}<\infty, \quad \limsup _{\|\mathbf{u}\| \rightarrow \infty} \frac{f_{i}(\mathbf{u})}{\|\mathbf{u}\|^{p-1}}<\infty,
$$

where $\mathbf{u}=\left(u_{1}, \ldots, u_{n}\right) \in \mathbb{R}_{+}^{n}$. 
Theorem 1.3. Assume (H1) and (H4) hold. Then there is an $R_{0}>0$ such that (1.1) has no positive solution for $0<R<R_{0}$.

We now give two examples to demonstrate the theorems.

Example 1.4.

$$
\begin{gathered}
\operatorname{div}\left(\left|\nabla u_{1}\right|^{p-2} \nabla u_{1}\right)+e^{\left(u_{1}+\cdots+u_{n}\right)}=0 \quad \text { in } B, \\
\operatorname{div}\left(\left|\nabla u_{i}\right|^{p-2} \nabla u_{i}\right)+f_{i}\left(u_{1}, \ldots, u_{n}\right) \quad \text { in } B, i=2, \ldots, n, \\
u_{i}=0 \quad \text { on } \partial B, i=1, \ldots, n,
\end{gathered}
$$

where $p>1, B=\left\{x \in \mathbb{R}^{N}:|x|<R\right\}, R>0, f_{i}$ are any nonnegative continuous functions. Then (1.10) has a positive solution for sufficiently small $R>0$ according to Theorem 1.1.

Example 1.5.

$$
\begin{gathered}
\operatorname{div}\left(\left|\nabla u_{i}\right|^{p-2} \nabla u_{i}\right)+\left(u_{1}+\cdots+u_{n}\right)^{p_{i}}=0 \quad \text { in } B i=1, \ldots, n, \\
u_{i}=0 \quad \text { on } \partial B, i=1, \ldots, n,
\end{gathered}
$$

where $p>1,0<p_{1}, p_{2}, \ldots, p_{n}<p-1, B=\left\{x \in \mathbb{R}^{N}:|x|<R\right\}, R>0$. Then (1.11) has a nontrivial solution for all $R>0$ according to Theorem 1.2.

\section{Preliminaries}

Let $\varphi(t)=|t|^{p-2} t$, then, for $t>0, \varphi(t)=t^{p-1}$ and $\varphi^{-1}(t)=t^{1 /(p-1)}$. It is easy to see that $\varphi^{-1}(\sigma \varphi(t))=\varphi^{-1}(\sigma) t$ for $t>0$ and $\sigma>0$.

We will deal with classical solutions of (1.1), namely a vector-valued function $\mathbf{u}=$ $\left(u_{1}(t), \ldots, u_{n}(t)\right)$ with $u_{i} \in C^{1}[0, R]$, and $\varphi\left(u_{i}^{\prime}\right) \in C^{1}(0, R), i=1, \ldots, n$, which satisfies $(1.1)$. A solution $\mathbf{u}(t)=\left(u_{1}(t), \ldots, u_{n}(t)\right)$ is positive if $u_{i}(t) \geq 0, i=1, \ldots, n$, for all $t \in(0, R)$ and there is at least one nontrivial component of $\mathbf{u}$. In fact, it is easy to prove that such a nontrivial component of $\mathbf{u}$ is positive on $(0, R)$.

Applying the change of variables, $t=R r$, we can transform (1.1) into the form

$$
\begin{gathered}
\left(r^{N-1} \varphi\left(\frac{u_{i}^{\prime}(r)}{R}\right)\right)^{\prime}+R r^{N-1} f_{i}(\mathbf{u})=0, \quad 0<r<1, i=1, \ldots, n, \\
\mathbf{u}^{\prime}(0)=\mathbf{u}(1)=0 .
\end{gathered}
$$

Note that we still use $u_{i}(r)$ and $v_{i}(r)$ for the new functions, $u_{i}(R r)$ and $v_{i}(R r)$. Thus $d u_{i}(t) / d t=\left(d u_{i}(R r) / d r\right)(d r / d t)=\left(d u_{i}(R r) / d r\right)(1 / R)=\left(d u_{i}(r) / d r\right)(1 / R)$.

We now recall some concepts and conclusions on the fixed point index in a cone in $[2,3]$. Let $X$ be a Banach space and let $K$ be a closed, nonempty subset of $X$. $K$ is said to be a cone if (i) $\alpha u+\beta v \in K$ for all $u, v \in K$ and all $\alpha, \beta>0$ and (ii) $u,-u \in K$ imply $u=0$. Assume $\Omega$ is a bounded open subset in $X$ with the boundary $\partial \Omega$, and let $T: K \cap \bar{\Omega} \rightarrow K$ be completely continuous such that $T x \neq x$ for $x \in \partial \Omega \cap K$, then the fixed point index $i(T, K \cap \Omega, K)$ is defined. If $i(T, K \cap \Omega, K) \neq 0$, then $T$ has a fixed point in $K \cap \Omega$. The following well-known result of the fixed point index is crucial in our arguments. 
4 Existence and nonexistence of positive solutions

Lemma $2.1[2,3]$. Let $E$ be a Banach space and $K$ a cone in E. Further let $r>0, K_{r}=$ $\{u \in K:\|x\|<r\}$, and $\partial K_{r}=\{u \in K:\|x\|=r\}$. Assume that $T: \bar{K}_{r} \rightarrow K$ is completely continuous.

(i) If there exists an $x_{0} \in K \backslash\{0\}$ such that

$$
x-T x \neq t x_{0} \quad \forall x \in \partial K_{r}, t \geq 0,
$$

then

$$
i\left(T, K_{r}, K\right)=0 \text {. }
$$

(ii) If $\|T x\| \leq\|x\|$ for $x \in \partial K_{r}$ and $T x \neq x$ for $x \in \partial K_{r}$, then

$$
i\left(T, K_{r}, K\right)=1 \text {. }
$$

In order to apply Lemma 2.1 to $(1.1)$, let $X$ be the Banach space $\underbrace{C[0,1] \times \cdots \times C[0,1]}$ and, for $\mathbf{u}=\left(u_{1}, \ldots, u_{n}\right) \in X$,

$$
\|\mathbf{u}\|=\sum_{i=1}^{n} \sup _{t \in[0,1]}\left|u_{i}(t)\right| .
$$

For $\mathbf{u} \in X$ or $\mathbb{R}_{+}^{n},\|\mathbf{u}\|$ denotes the norm of $\mathbf{u}$ in $X$ or $\mathbb{R}_{+}^{n}$, respectively.

Define $K$ to be a cone in $X$ defined by

$$
K=\left\{\left(u_{1}, \ldots, u_{n}\right) \in X: u_{i}(t) \geq 0, t \in[0,1], i=1, \ldots, n\right\} .
$$

Also, for each $r$ positive number, define $\Omega_{r}$ by

$$
\Omega_{r}=\{\mathbf{u} \in K:\|\mathbf{u}\|<r\} .
$$

Note that $\partial \Omega_{r}=\{\mathbf{u} \in K:\|\mathbf{u}\|=r\}$.

Let $\mathbf{T}: K \rightarrow X$ be a map with components $\left(T^{1}, \ldots, T^{n}\right)$. We define $T^{i}, i=1, \ldots, n$, by

$$
T^{i} \mathbf{u}(t)=R \int_{t}^{1} \varphi^{-1}\left(\frac{R}{s^{N-1}} \int_{0}^{s} \tau^{N-1} f_{i}(\mathbf{u}(\tau)) d \tau\right) d s, \quad t \in[0,1] .
$$

It is straightforward to verify that the problem of finding positive solutions to (1.1) is equivalent to the fixed point equation

$$
\mathbf{T u}=\mathbf{u} \quad \text { in } K
$$

It is easy to show that $\mathbf{T}(K) \subset K$ and is completely continuous. In particular, we have the following assertion.

Lemma 2.2. Assume (H1) holds. Then $\mathbf{T}(K) \subset K$ and $\mathbf{T}: K \rightarrow K$ is completely continuous.

For each $i=1, \ldots, n$, define new function $\hat{f}_{i}(t): \mathbb{R}_{+} \rightarrow \mathbb{R}_{+}$by

$$
\hat{f}_{i}(t)=\max \left\{f_{i}(\mathbf{u}): \mathbf{u} \in \mathbb{R}_{+}^{n} \text { and }\|\mathbf{u}\| \leq t\right\} .
$$


Lemma 2.3 [7, Lemma 2.8]. Let (H1) hold and assume $\lim _{\|\mathbf{u}\|} \rightarrow \infty\left(f_{i}(\mathbf{u}) /\|\mathbf{u}\| p^{p-1}\right)=f_{\infty}^{i}$ and $\lim _{\|\mathbf{u}\| \rightarrow 0}\left(f_{i}(\mathbf{u}) /\|\mathbf{u}\|^{p-1}\right)=f_{0}^{i}, \mathbf{u} \in \mathbb{R}_{+}^{n}, f_{0}^{i}, f_{\infty}^{i} \in[0, \infty]$ for some $i \in\{1, \ldots, n\}$.

Then $\lim _{t \rightarrow 0^{+}}\left(\hat{f}_{i}(t) / \varphi(t)\right)=f_{0}^{i}$ and $\lim _{t \rightarrow \infty}\left(\hat{f}_{i}(t) / \varphi(t)\right)=f_{\infty}^{i}$.

Lemma 2.4. Assume (H1) holds and let $r>0$. If there exists an $\varepsilon>0$ such that

$$
\hat{f}_{i}(r) \leq \varphi(\varepsilon) \varphi(r), \quad i=1, \ldots, n,
$$

then

$$
\|\mathbf{T u}\| \leq n R \varphi^{-1}\left[\frac{R}{N}\right] \varepsilon\|\mathbf{u}\| \quad \text { for } \mathbf{u} \in \partial \Omega_{r}
$$

Proof. From the definition of $T$, for $\mathbf{u} \in \partial \Omega_{r}$, we have

$$
\begin{aligned}
\|\mathbf{T u}\| & =\sum_{i=1}^{n} \sup _{t \in[0,1]}\left|T^{i} \mathbf{u}(t)\right|=R \sum_{i=1}^{n} \int_{0}^{1} \varphi^{-1}\left[\frac{R}{s^{N-1}} \int_{0}^{s} \tau^{N-1} f_{i}(\mathbf{u}(\tau)) d \tau\right] d s \\
& \leq R \sum_{i=1}^{n} \int_{0}^{1} \varphi^{-1}\left[\frac{R}{s^{N-1}} \int_{0}^{s} \tau^{N-1} d \tau \hat{f}_{i}(r)\right] d s \leq n R \varphi^{-1}\left[\frac{R}{N} \varphi(\varepsilon) \varphi(r)\right] \\
& =n R \varphi^{-1}\left[\frac{R}{N} \varphi(\varepsilon r)\right]=n R \varphi^{-1}\left(\frac{R}{N}\right) \varepsilon\|\mathbf{u}\| .
\end{aligned}
$$

Lemma 2.5. Assume (H1) holds and $r>0$. Then

$$
\|\mathbf{T u}\| \leq n R \varphi^{-1}\left(\frac{R}{N}\right) \varphi^{-1}\left(\widehat{M}_{r}\right) \quad \text { holds } \forall \mathbf{u} \in \partial \Omega_{r}
$$

where $\widehat{M}_{r}=1+\max \left\{f_{i}(\mathbf{u}): \mathbf{u} \in \mathbb{R}_{+}^{n}\right.$ and $\left.\|\mathbf{u}\| \leq r, i=1, \ldots, n\right\}>0$.

Proof. Since $f_{i}(\mathbf{u}(t)) \leq \widehat{M}_{r}=\varphi\left(\varphi^{-1}\left(\widehat{M}_{r}\right)\right)$ for $t \in[0,1], i=1, \ldots, n$, it is easy to see that this lemma can be shown in a similar manner as Lemma 2.4 .

\section{Proof of Theorem 1.1}

Fix a number $r_{2}>0$. Lemma 2.5 implies that there exists an $R_{0}>0$ such that

$$
\|\mathbf{T u}\|<\|\mathbf{u}\| \quad \text { for } \mathbf{u} \in \partial \Omega_{r_{2}}, \quad 0<R<R_{0} .
$$

Now let $0<R<R_{0}$ and $\eta>0$ be such that

$$
R \frac{\eta}{2} \varphi^{-1}\left(\frac{R}{N 4^{N}}\right) \geq 1
$$


6 Existence and nonexistence of positive solutions

Since

$$
\lim _{\|\mathbf{u}\| \rightarrow 0} \frac{f_{i}(\mathbf{u})}{\|\mathbf{u}\| p^{p-1}}=\infty
$$

there is $0<r_{1}<r_{2}$ such that

$$
f_{i}(\mathbf{u}) \geq \varphi(\eta) \varphi(\|\mathbf{u}\|)
$$

for $\mathbf{u}=\left(u_{1}, \ldots, u_{n}\right) \in \mathbb{R}_{+}^{n}$ and $\|\mathbf{u}\| \leq r_{1}$.

If $\mathbf{u}-\mathbf{T u}=0$ for some $\mathbf{u} \in \partial \Omega_{r_{1}}$, we already find the desired solution of (1.1). Therefore we assume that

$$
\mathbf{u}-\mathbf{T u} \neq 0 \quad \forall \mathbf{u} \in \partial \Omega_{r_{1}}
$$

we now claim that

$$
\mathbf{u}-\mathbf{T u} \neq t \mathbf{v} \quad \forall \mathbf{u} \in \partial \Omega_{r_{1}}, t \geq 0,
$$

where $\mathbf{v}=(\theta(r), \ldots, \theta(r))$, and $\theta \in C[0,1]$ such that $0 \leq \theta(r) \leq 1$ on $[0,1], \theta(r) \equiv 1$ on $[0,1 / 4]$ and $\theta(r) \equiv 0$ on $[1 / 2,1]$. Thus, $\mathbf{v} \in K \backslash\{0\}$. If there exists $\mathbf{u}^{*}=\left(u_{1}^{*}, \ldots, u_{n}^{*}\right) \in \partial \Omega_{r_{1}}$ and $t_{0} \geq 0$ such that $\mathbf{u}^{*}-\mathbf{T u}^{*}=t_{0} \mathbf{v}$, we will show that this leads to a contradiction. Since (3.5) is true, we have $t_{0}>0$. Since $\mathbf{T}(K) \subset K$, we obtain $u_{i}^{*}(r) \geq t_{0} \theta(r)$ for all $r \in[0,1]$. Let

$$
t^{*}=\sup \left\{t: u_{i}^{*}(r) \geq t \theta(r) \forall r \in[0,1]\right\} .
$$

It follows that $t_{0} \leq t^{*}<\infty$ and $u_{i}^{*}(r) \geq t^{*} \theta(r)$ for all $r \in[0,1]$. Now, for $r \in[0,1]$, we have

$$
\begin{aligned}
u_{i}^{*}(r) & =\mathbf{T}^{i} \mathbf{u}^{*}(r)+t_{0} \theta(r) \\
& =R \int_{r}^{1} \varphi^{-1}\left(\frac{R}{s^{N-1}} \int_{0}^{s} \tau^{N-1} f_{i}\left(\mathbf{u}^{*}(\tau)\right) d \tau\right) d s+t_{0} \theta(r) .
\end{aligned}
$$

Note that $\sum_{j=1}^{n} u_{j}^{*}(r) \leq r_{1}$ for $r \in[0,1]$. Formula (3.4) implies that, for $r \in[0,1 / 2]$,

$$
\begin{aligned}
u_{i}^{*}(r) & \geq R \int_{1 / 2}^{1} \varphi^{-1}\left(\frac{R}{s^{N-1}} \int_{0}^{s} \tau^{N-1} \varphi(\eta) \varphi\left(\sum_{j=1}^{n} u_{j}^{*}(\tau)\right) d \tau\right) d s+t_{0} \theta(r) \\
& \geq R \int_{1 / 2}^{1} \varphi^{-1}\left(R \int_{0}^{s} \tau^{N-1} \varphi(\eta) \varphi\left(u_{i}^{*}(\tau)\right) d \tau\right) d s+t_{0} \theta(r) \\
& \geq \frac{R}{2} \varphi^{-1}\left(R \int_{0}^{1 / 4} \tau^{N-1} \varphi(\eta) \varphi\left(t^{*} \theta(\tau)\right) d \tau\right)+t_{0} \theta(r) \\
& =\frac{R}{2} \varphi^{-1}\left(R \int_{0}^{1 / 4} \tau^{N-1} d \tau \varphi(\eta) \varphi\left(t^{*}\right)\right)+t_{0} \theta(r) \\
& =\frac{R}{2} \varphi^{-1}\left(\frac{R}{N 4^{N}} \varphi\left(\eta t^{*}\right)\right)+t_{0} \theta(r) .
\end{aligned}
$$


Now, in view of the fact that $\varphi^{-1}(\sigma \varphi(t))=\varphi^{-1}(\sigma) t$, we have, for $r \in[0,1 / 2]$,

$$
u_{i}^{*}(r) \geq t^{*} \frac{\eta R}{2} \varphi^{-1}\left(\frac{R}{N 4^{N}}\right)+t_{0} \theta(r) \geq t^{*}+t_{0} \theta(r) \geq\left(t^{*}+t_{0}\right) \theta(r)
$$

and hence

$$
u_{i}^{*}(r) \geq\left(t^{*}+t_{0}\right) \theta(r), \quad r \in[0,1],
$$

which is a contradiction to the definition of $t^{*}$. Thus, in view of Lemma 2.1,

$$
i\left(\mathrm{~T}, \Omega_{r_{1}}, K\right)=0, \quad i\left(\mathrm{~T}, \Omega_{r_{2}}, K\right)=1 .
$$

It follows from the additivity of the fixed point index that $i\left(\mathbf{T}, \Omega_{r_{2}} \backslash \bar{\Omega}_{r_{1}}, K\right)=1$. Thus, T has a fixed point in $\Omega_{r_{2}} \backslash \bar{\Omega}_{r_{1}}$, which is the desired positive solution of (1.1).

\section{Proof of Theorem 1.2}

Let $R$ be an arbitrary positive number. Since (H3) is true, it follows from Lemma 2.3 that $\lim _{t \rightarrow \infty}\left(\hat{f}_{i}(t) / \varphi(t)\right)=0, i=1, \ldots, n$. Hence, there is an $r_{2}>0$ such that

$$
\hat{f}_{i}\left(r_{2}\right) \leq \varphi(\varepsilon) \varphi\left(r_{2}\right), \quad i=1, \ldots, n,
$$

where the constant $\varepsilon>0$ satisfies

$$
n R \varphi^{-1}\left(\frac{R}{N}\right) \varepsilon<1
$$

Thus, we have by Lemma 2.4 that

$$
\|\mathbf{T}(\mathbf{u})\| \leq n R \varphi^{-1}\left(\frac{R}{N}\right) \varepsilon\|\mathbf{u}\|<\|\mathbf{u}\| \quad \text { for } \mathbf{u} \in \partial \Omega_{r_{2}} .
$$

By Lemma 2.1,

$$
i\left(\mathrm{~T}, \Omega_{r_{2}}, K\right)=1 \text {. }
$$

Next using exactly the same argument as in Theorem 1.1, we can determine a $0<r_{1}<r_{2}$ from (H2) such that (3.6) holds. Note that $R$ can be any positive number for Theorem 1.2. Thus it follows from Lemma 2.1 that

$$
i\left(\mathrm{~T}, \Omega_{r_{1}}, K\right)=0, \quad i\left(\mathrm{~T}, \Omega_{r_{2}}, K\right)=1,
$$

and hence, $i\left(\mathbf{T}, \Omega_{r_{2}} \backslash \bar{\Omega}_{r_{1}}, K\right)=1$. Thus, $\mathbf{T}$ has a fixed point in $\Omega_{r_{2}} \backslash \bar{\Omega}_{r_{1}}$. Consequently, (1.1) has a positive solution for all $R>0$. 
8 Existence and nonexistence of positive solutions

\section{Proof of Theorem 1.3}

Since (H4) is true, for each $i=1, \ldots, n$, there exist positive numbers $\varepsilon_{1}^{i}, \varepsilon_{2}^{i}, r_{1}^{i}$, and $r_{2}^{i}$ such that $r_{1}^{i}<r_{2}^{i}$,

$$
\begin{array}{ll}
f_{i}(\mathbf{u}) \leq \varepsilon_{1}^{i} \varphi(\|\mathbf{u}\|) & \text { for } \mathbf{u} \in \mathbb{R}_{+}^{n},\|\mathbf{u}\| \leq r_{1}^{i}, \\
f_{i}(\mathbf{u}) \leq \varepsilon_{2}^{i} \varphi(\|\mathbf{u}\|) & \text { for } \mathbf{u} \in \mathbb{R}_{+}^{n},\|\mathbf{u}\| \geq r_{2}^{i} .
\end{array}
$$

Let

$$
\varepsilon^{i}=\max \left\{\varepsilon_{1}^{i}, \varepsilon_{2}^{i}, \max \left\{\frac{f_{i}(\mathbf{u})}{\varphi(\|\mathbf{u}\|)}: \mathbf{u} \in \mathbb{R}_{+}^{n}, r_{1}^{i} \leq\|\mathbf{u}\| \leq r_{2}^{i}\right\}\right\}>0
$$

and $\varepsilon=\max _{i=1, \ldots, n}\left\{\varepsilon^{i}\right\}>0$. Thus, we have

$$
f_{i}(\mathbf{u}) \leq \varepsilon \varphi(\|\mathbf{u}\|) \quad \text { for } \mathbf{u} \in \mathbb{R}_{+}^{n}, i=1, \ldots, n .
$$

Assume $\mathbf{v}(t)$ is a positive solution of (1.1). We will show that this leads to a contradiction for $0<R<R_{0}$, where

$$
n R_{0} \varphi^{-1}\left(\frac{R_{0} \varepsilon}{N}\right)<1
$$

In fact, for $0<R<R_{0}$, since $\operatorname{Tv}(t)=\mathbf{v}(t)$ for $t \in[0,1]$, we find

$$
\begin{aligned}
\|\mathbf{v}\| & =\|\mathbf{T} \mathbf{v}\|=\sum_{i=1}^{n} \sup _{t \in[0,1]}\left|T^{i} \mathbf{v}(t)\right| \leq R \sum_{i=1}^{n} \int_{0}^{1} \varphi^{-1}\left[\frac{R}{s^{N-1}} \int_{0}^{s} \tau^{N-1} d \tau \varepsilon \varphi(\|\mathbf{v}\|)\right] d s \\
& \leq n R \varphi^{-1}\left(\frac{R \varepsilon}{N} \varphi(\|\mathbf{v}\|)\right)=n R \varphi^{-1}\left(\frac{R \varepsilon}{N}\right)\|\mathbf{v}\|<\|\mathbf{v}\|,
\end{aligned}
$$

which is a contradiction.

\section{Acknowledgments}

The author thanks the three reviewers for their comments to improve the presentation of the paper.

\section{References}

[1] R. Dalmasso, Existence and uniqueness of positive solutions of semilinear elliptic systems, Nonlinear Analysis 39 (2000), no. 5, 559-568.

[2] K. Deimling, Nonlinear Functional Analysis, Springer, Berlin, 1985.

[3] D. Guo and V. Lakshmikantham, Nonlinear Problems in Abstract Cones, Notes and Reports in Mathematics in Science and Engineering, vol. 5, Academic Press, Massachusetts, 1988.

[4] M. A. Krasnosel'skili, Positive Solutions of Operator Equations, Noordhoff, Groningen, 1964.

[5] P.-L. Lions, On the existence of positive solutions of semilinear elliptic equations, SIAM Review 24 (1982), no. 4, 441-467.

[6] D. O’Regan and H. Wang, Positive solutions for p-laplacian systems in a ball, in preparation. 
[7] H. Wang, On the number of positive solutions of nonlinear systems, Journal of Mathematical Analysis and Applications 281 (2003), no. 1, 287-306.

[8] An existence theorem for quasilinear systems, to appear in Proceedings of the Edinburgh Mathematical Society.

Haiyan Wang: Department of Mathematical Sciences \& Applied Computing, Arizona State University, Phoenix, AZ 85069-7100, USA

E-mail address: wangh@asu.edu 